\title{
Aggregation-based rapid detection of Enterovirus 71 using surface- enhanced Raman spectroscopy (Withdrawal Notice)
}

Miguel Reyes, Shuai He, Marek Piotrowski, Swee Kim Ang, Jingqi Chan, et al.

Miguel Reyes, Shuai He, Marek Piotrowski, Swee Kim Ang, Jingqi Chan, Justin Jang Hann Chu, James Chen Yong Kah, "Aggregation-based rapid detection of Enterovirus 71 using surface-enhanced Raman spectroscopy (Withdrawal Notice)," Proc. SPIE 10251, Biomedical Imaging and Sensing Conference, 102511C (17 May 2017); doi: 10.1117/12.2269391

Event: SPIE Technologies and Applications of Structured Light, 2017, Yokohama, Japan 


\section{Aggregation-based rapid detection of Enterovirus 71 using surface-enhanced Raman spectroscopy (withdrawal notice)}

Proc. SPIE 10251, 102511C (2017)

Online Publication Date: 18 April 2017

Withdrawn from Publication: 10 May 2017

Conference Date: 19-21 April 2017

Conference Location: Yokohama, Japan

Conference Title: Biomedical Imaging and Sensing conference

Conference Chairs: T. Yatagai, Y. Aizu, O. Matoba, and Y. Awatsuji

Miguel Reyes, Marek Piotrowski, Swee Kim Ang, Jingqi Chan, Shuai He, Justin Jang Hann Chu, James Chen Yong Kah

National Univ. of Singapore (Singapore); Jerzy Haber Institute of Catalysis and Surface Chemistry (Poland); Temasek Junior College (Singapore)

This paper has been withdrawn by the publisher because it was not presented at the conference. 